was obliged to use too high a power, not having a suitable eye piece."

In conclusion, I wish most emphatically to fay and place publically on record, knowing that probably many years, perhaps centuries may roll by, before my observations can be verified, that $I$ have not been the victim of a delusion, nor have $I$ attempted to palm off upon the world a deception. Scarcely a clear night in 22 years has passed, that my telescope has not been pointed to the sky, searching out the mysteries that lie within its grasp, yet not once has it deceived me, nor did it them.

I know that no such configuration of stars, nor no two of the same magnitude exist there, and, astronomers are left no alteruative but to conclude that $I$ saw at least one, and probably two intra-Mercurial planets, or, that I saw nothing whatever.

My observation can admit of but 3 interpretations, viz. $18^{\mathfrak{2}}$ that $I$ saw $\theta$, and a new object which Watson also saw 5 minutes previously, his planet $(a)$, or, $2^{\text {nd }}$, that I saw $\theta$ and a new body $7^{\circ}$ from it, which he did not see, or, $3^{\mathrm{d}}$, that $I$ did not see $\theta$ at all, but two new objects near by, which he did not see.

The first two are improbable in the highest degree, for, how could Watson, who says he saw $\theta$ have also

Rochester N. Y., July 41879. failed to see another object equally bright, but $\tau^{\prime}$ away when the size of his field was about 1"? Again, how could $I$ if one was $\theta$, have failed to see planet $(a)$ but 72 ' away, and half a magnitude brighter, when my field was 90', and telescope half an inch the larger?

Prof. Peters' attempt to ignore my observations of two new objects, seen unexpectedly, and of course without bias, three different times, and in the same field, will weigh nothing with astronomers, who, without prejudice are seeking for truth.

Knowing well he had no rantage ground of attack, that he was powerless to combat them with errors of cal. culation, or of circle reading, or the inaccuracy of pencil marking etc. as he has attempted to do with Prof. Watson's, observations he chose not to discuss mine at all, a wise thing for him to do.

Why need it be considered impossible that there are planets nearer the sun than Mercury, especially when the advance of the perihelion of his orbit seems to demand them.

That they may be as numerous as those between Mars and Jupiter, no man can deny, and their discovery would only add another to the many wonderful things in which astronomy deals so largely.

Lewis Switt.

\title{
Ueber den Erfinder des negativen Oculars.
}

Bekanntlich wird die Erfindung des negativen Oculars von einigen Christian Huygens, von anderen Campani zugeschrieben. Indem ich in diesem Sommer einen kleinen Aufsatz über Fernröhre behufs einer neuen, von Herrn Marine-Lieutenant E. Simon van der Aa besorgten, Ausgabe von "Brouwer's Zeevaartkunde" zusammenstellte, wobei ich auch, wo es vorkam, auf das Geschichtliche Rücksicht nahm, wollte ich untersuchen, welcher von diesen beiden, Campani oder Huygens, eigentlich als der Urheber des negativen Oculars anzusehen ist. Es hat sich dabei ergeben, dass die verschiedenen Schriftsteller, indem sie versäumten, die Quellen selbst zu rathpflegen, unrichtige Vorstellungen von einander übernommen haben, so dass am Ende allerhand unwahre Legenden über das negative Ocular current geworden sind.

Christian Huygens hat sein Ocular in seiner Dioptrica beschrieben, welche erst nach seinem Tode von
Fullenius und de Volder in den Opusculis posthumis, Lugd. Batar. 1703, herausgegeben ist; die 20 Ed. ist ron Amsterdam, 1720.

Die Propositio LI dieses Werkes, worin das Doppelocular beschrieben wird, lautet so: "Quomodo, pro duabus convexis tria adhibendo amplior fiat telescopii prospectus, quo, ad sidera spectanda, utimur."

Durch die duo convexa werden hier Objectiv und einfaches Ocular verstanden; wird nur ein Doppelocular in die Stelle gesetzt, so werden drei Gläser angewandt, und das Feld (telescopii prospectus) wird grösser.

$\mathrm{Zu}$ dieser Propositio gehört eine Figur, Tab. XVI, Fig. 6, wo nur biconvexe linsen gezeichnet sind, und nicht plan-convexe, wie so oft behauptet wird, dass Chr. Huygens schon angewandt hat. Es heisst darüber wie folgt:

Sit ratio augmenti proposita ea quae $P$ ad $Q$. Lens exterior $L$, focus ejus $G$. Et ut $P$ ad $Q$, ita sit $L G$ 
ad $G K$, cadente puncto $K$ inter $L$ et $G$. Et in $K$ lens convexa statuatur, cujus foci distantia $K V$ sit tripla ad $K G$, et divisa $K V$ aequaliter in $S$ statuatur ibi lens altera $E E$, cujus foci distantia $S H$ fit $\frac{1}{2} S K$. Oculus vero sit in $M$, posita $S M$ distantia $2 \mid 2 \frac{1}{2} K G$. Erit factum quod quaeritur. (Das Zeichen $2 \mid 2$ bedeutet $=$ ).

Die hierzu gehörende Figur lässt sich nach dem Text leicht zeichnen. Nennt man

die Brennweite des Objectivs ...........F,

die Vergrösserung des Fernrohrs ........... n,

die Brennweite der Collectivlinse ..........f,

die Entfernuug der beiden Ocularlinsen .......d,

die Brennweite der Augenlinse $. . . . \ldots \ldots . . . f^{\prime}$,

den Abstand des Ortes des Auges von der

Augenlinse .................... $a$,

so ist:

$$
\begin{aligned}
f^{\prime} & =3 \frac{F^{\prime}}{n}=4 f^{\prime \prime}, \\
d & =\frac{3}{2} \frac{F}{n}=2 f^{\prime}, \\
f^{\prime} & =\frac{3}{4} \frac{F}{n}, \\
a & =\frac{1}{2} \frac{F}{n},
\end{aligned}
$$

die Brennweite des aequivalenten Oculars richtig

$$
\frac{t f^{\prime \prime}}{f+f^{\prime}-d}=\frac{F^{\prime}}{n} \text {. }
$$

Von plan-convexen Linsen ist in der Dioptrica von Huygens bei den Ocularen gar keine Rede, und eben so wenig von einem Ocular, wo das Verhältniss

$$
f^{\prime}: d: f^{\prime}=3: 2: 1
$$

ist.

Im Systema Saturnium, das im J. 1659 gedruckt worden ist, findet man über das Fernrohr, womit Huygens den Saturnsmond entdeckt hat, das folgende;

"Sed antequam observationes exhibeamus, de telescopiis nostris pauca referre expediet, ... Primus quem adhibuimus duodenos pedes non excedebat*) duobis convexis vitris instructus, quorum id quod oculo vicinum erat, radios parallelos cogebat at trium paulo minus pollicum sive unciarum pedis Rhenolandici distantiam. Ea planetam novum et deteximus primum et per aliqnot menses observavimus nec non formam eam Saturni quae a nemine adhuc precepta fuerat quamque postea describemus, licet non prorsus erroris expertem." (Planeta

*) Aus den Handschriften von Chr. Huygens hat Kaiser schon 1846 gefunden, dass die Brennweite des hier genannten Fernrohrs nicht grösser als to Fuss war. novus soll wohl der Satellit heissen, also sind beide noch mit einem Keppleriani'schen Fernrohre von Huygens beobachtet:)

"Inde vero duplicata priori longitudine, simul duplo proprioribus sideribus facti sumus, multoque melius faciliusque phaenomena omnia adnotavimus. Et hi quidem tubi 23 pedum, e ferri bractea constructi sunt, habentque ab altera parte vitrum insertum, cujus latitudo ad quatuor pollices, sed in quo non major pateat circulus quam diametro duorum pollicum cum triente. Ab altera parte, quae nimirum oculo admovetur, bina sunt vitra minora, $1 \frac{1}{2}$ pollicem diametro aequantia, juncta invicem, quaeque hoc pacto aequipollent convexo colligenti radios parallelos ad intervallum unciarum 3 aut paulo etiam angustius. - ..... Centuplem itaque fere rationem hanc in perspicillis nostris esse constat, cum Galileana non ultra trigecuplam processerint....."

Es erhellt hieraus, dass die Dioptrica im J. 16509 entweder schon geschrieben oder dass wenigstens der Verfasser schon damit beschäftigt war; die Beobachtungen, welche im Systema Saturnium beschrieben werden, fangen am 25. März 1650 an, und das 23 füss. Fernrohr wurde am 19. Februar 1656 zuerst angewandt, dies kann also wahrscheinlich auch für das Datum gelten, wo Huygens zuerst sein Doppelocular benutzt hat.

Gehen wir nun zu Campani über. In seinem Biographischen Wörterbuche führt Poggendorf an:

Campani Giuseppi...... schrieb: Raggualgio di due nuove osservasioni, una celeste in ordine alla stella di Saturno, e terrestre l' altra in ordine a gl' instrumenti Roma 1664, 1665. ..... Account of the improvement of optich glasses (Phil. Tr. 1665). -

In der hiesigen Bibliothek sind die italienisehen Bücher überhaupt schlecht vertreten, und der Raggualgio etc. ist auch nicht da. Der genannte Jahrgang ist der erste der Phil. Transactions, und der Account, welcher laut des "Epistle dedicatory" an die Royal Society, von Oldenburg redigirt ist, ist der erste Artikel der ersten Nummer, und trägt das Datum March 6, $166 \frac{1}{5}$. Oldenburg hat seine Kenntniss von Campani's Leistungen nicht aus dem Original, sondern aus einem französischen Berichte bekommen und sagt darüber das Folgende:

"The first (sc. of the particulars) regardeth the excellency of the long Telescopes made by the said Campani, who pretends to have found a way to work great Optich Glasses with a Turne-tool, without any mould: "And whereas hitherto it has been found by Experience, that small Glasses are in proportion better to see with, upon the Earth, then the great 
ones; that Auther affirms, that his are equally good for the Earth, and for making Observations in the Heavens. Besides, he useth three Eye-Glasses for his great $\mathrm{Te}$ lescopes, without finding any Iris or such Rainbow colors, as do usually appear in ordinary Glasses, and prove an impediment to Observations."

Dies war nicht sehr geeignet die Frage zu lösen, denn von einem Ocular aus zwei Linsen kein Wort, wohl von einem aus drei Linsen.

Um doch über die Ansprüche des Campani Näheres zu vernehmen, gab ich mir die Freiheit, Prof Schiaparelli zu bitten, wo möglich das ursprüngliche Werk von Campani nachzusehen, hierauf erhielt ich die nachfolgende Antwort:

$$
\text { "Milan, le 9. Août } 1879 .
$$

A l'égard du premier inventeur de l'oculaire dit négatif, j’ai du diftérer ma réponse de quelques jours; n'ayant pu trouver dans les Bibliothèques de Milan aucun exemplaire du "Ragguaglio" de Campani j'ai obtenu de me la faire arriver de Rome. Je trouve dans cet opuscule le trait suivant, qui me parait décider la question (pag. 37):

In proposito di questi Cannocchiali di straordinaria lunghezza, mi pare di dover lodare il pensiero del Signor Christiano Hugenii (Sic) il quale nelle sue osservazioni di Saturno, stampate l'anno 1659 si servi (come ivi riferisce a carte 4) in luogo della semplice lente oculare tutta convessa, d'una lente composta di due retri piano-convessi, a fin de sfuggire in questa maniera alcuni difetti, che nel vetro di straordinaria grossezza sono quasi inevitabili.

Voilà Campani lui-même qui déclare que l'oculaire employé par M. Huygens n'est pas de sa propre invention, mais qu'il en apprend la construction par le Systema Saturnium.

Quel était done le but des trois verres dans les oculaires de Campani? C'était simplement d'en faire des oculaires terrestres. $\mathrm{Je}$ trouve dans le même livre cet autre passage (p. 23):

E questo ultimo eannocchiale palmi 55 lungo, di 4 vetri: ma con due arteficii che possono dirsi di mia invenzioni. Il primo é che etiandio con la mutazione di due altri oggettivi che ci ho fabbricati uno di palmi 52 e di 50 l'altro, senza variar la distanza dell' occhio dalla lente occulare, restano tutti e tre i cannocchiali Astronomici, togliendo solo le due lenti de mezzo." L'altro etc.
Le Ragguaglio contient encore d'important un très beau dessin de Saturne.

Je vous prie monsieur, etc.

$$
\text { (Signé) J. V. Schiaparelli. }
$$

Woher nun Campani ableitete, dass Chr. Huygens plan-convexe Linsen anwandte, leuchtet nicht ein. Aus Huygens Schriften kann ich es nicht finden. Die Tafeln zeigen nur Oculare mit equiconvexen Gläsern und es liegt auf der Hand, dass wenn Huygens die Verwechslung mit plan-convexen Linsen beabsichtigte, er dies ohne $Z_{\text {weifel im }}$ Texte absichtlich erwähnt hätte.

Die Propositio LIV der Dioptrica hat die folgende Ueberschrift: Telescopii ex quatuor convexis compositi, constructionem explicare, quores visae erectae spectanturet magna copia.

Die hier gemeinte Construction ist die folgende:

$$
f^{\prime}=f^{\prime}=f^{*} \quad d=d^{\prime}=2 f
$$

Weiter sagt Huygens: Haec egregia lentium compositio Romae, nescio a quo, primum fuit inventa, multum tamen adjuta annulo seu diaphragmate quod ad $H$ loco medio inter lentes $E$ et $D$ (die $3^{\circ}$ und $2^{\circ}$ rom Objectiv ab gerechnet) vel ad $B$ focum communem lentium $A$ et $C$ (Objectiv und erste Linse) inseritur; cujus usum non ante cognitum explicuimus in libro de causis phaenoinenon Saturni.

Wo das Objectiv des 23 füssigen Fernrohrs, welches Huygens vom 19. Februar 1656 an anwandte, geblieben ist, ist, mir wenigstens, unbekannt. Das Objectiv des 10 füssigen, womit Huygens den Satelliten entdeckte und seine ersten Beobachtungen des Ringes anstellte, hat Prof. Harting im J. 1867 im hiesigen physikalischen Cabinet aufgefunden*), Oculare waren aber nicht dabei. Ein anderes Objectiv von Chr. Huygens von 12 Fuss Brennweite ist im J. 1846 von Kaiser im physikalischen Cabinet $\mathrm{zu}$ Leiden aufgefunden und in Nr. 592 dieser Zeitschrift beschrieben worden. Es war dabei aber das Rohr aus Eisenblech und ein dreifaches Ocular,

*) Prof. Harting hat diesen Fund in der holl. Zeitschrift „Album der Natuur", Jahrgang I 867 , p. 274 bekannt gemacht. Der Durchmesser des Objectivs ist 5,7 Centimeter; die Brennweite 3,17 Meter; es ist planconvex (aus einem Spiegel geschliffen) und hat eine Dicke" von 3,2 Millimeter. Nahe bei der Peripherie ist in kleiner Cursivschrift das Anagramm gravirt: Admovere ocalis distantia sidera nostris, aber ohne die zugehörenden Buchstaben (vvvvrvv cce $\operatorname{rrr} h \mathrm{~h} \mathrm{~b} \mathrm{q} x$ ) wie es Gravesande im Lebensberichte von Huygens vor den "Opera varia" L. B. I 824 irrig mittheilte. Dies Anagramm war bekanntlich ein Räthsel, den Fachgenossen vorläufig angeboten, dessen Lösung durch Umsetzung der Buchstaben erhalten werden musste, uud so lautete : Saturnus luna sua circumducitur sexdecim diebus horis quatuor. 
jedoch nioht genau von der oben angegebenen Construction bewahrt geblieben, *) welches sioh aber in ein Rohr mit zwei Glasern und ein Rohr mit dem dritten Glase trennen lasst. Aus dem Umstand, dass Kaiser weder a. a. O., noch in seiner, dem damaligen k. niederlandischen Institute angebotenen Abhandlung, die Art der Glaser beschreibt, kann man wohl schliessen, dass sie biconvex sind, wie ich ! mich auch erinnere sie fruher gesehen zu haben.

Ein aohtes hugenianisches Doppelocular ist auch in Leiden nicht aufgefunden.

Aus dem oben Mitgetheilten können, glaube ich, folgende Schlüsse abgeleitet werden:

1. Christian Huygens war der Erfinder des 80genannten negativen Doppeloculars.

2. Die Jahreszahl der Erfindung ist wahrscheinlich 1655, die erste Anwendung scheint am 19. Februar 1656 stattgefunden zu haben.

3. Das Verbaltniss $f, d, f^{\prime}$ war bei Huygens 4,2 , 1 und nicht 3, 2, 1, wie aligemein behauptet wird.

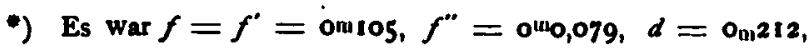
$d^{\prime}=\mathrm{om}_{182}, a=0$ ow058; Durchmesser $0.040,0.040$ und 0.038 , Dicken $0.0040,0.0048$ und 0.058 Meter.
4. Das Huygens'sche Ocular war also nicht achro. matisoh, denn hierfur sollte $d$ sehr nahe $=\mathrm{t}\left(f+f^{\prime}\right)$ sein, wie beim Verbaltnisse 3, 2, 1 wohl stattfindet, bei $4,2,1$ aber nicht.

5. So lange der Gegenbeweis nicht geliefert ist, muss man annehmen, dass Huygens fur seine Oculare equiconvexe Linsen benutzt hat.

Wer also bei der Construction der negativen Oculare zuerst das Verbultniss 3, 2, 1, und wer euerst die spater gebrauchlichen plan-converen Linsen eingefuhrt hat, - welche Combination ubrigens (soweit das dem Quadrat der halben Oeffnung proportionale Glied reicht) nach Airy's Analyse, (Cambr. Phil. Tr. Vol. III Part II p. 20) nicht besser ist als jene von zwoi biconvexen Linsen, - wurde noch nkher zu untersuchen sein.

6. Das Campani'sohe Ocular ist das aus drei Linsen bestehende, terrestrische Ocular, an dessen Stelle jetzt schon das Dollond'sche, aus vier Linsen zusammengesetzte, getreten ist.

Utrecht, 28. August 1879

J. A. C. Oudemans.

Observations of the Companion of Sirius.

(Communicated by Rear Admiral John Rodgers U. S. Nary, Superintendent Naval Observatory, Washington).

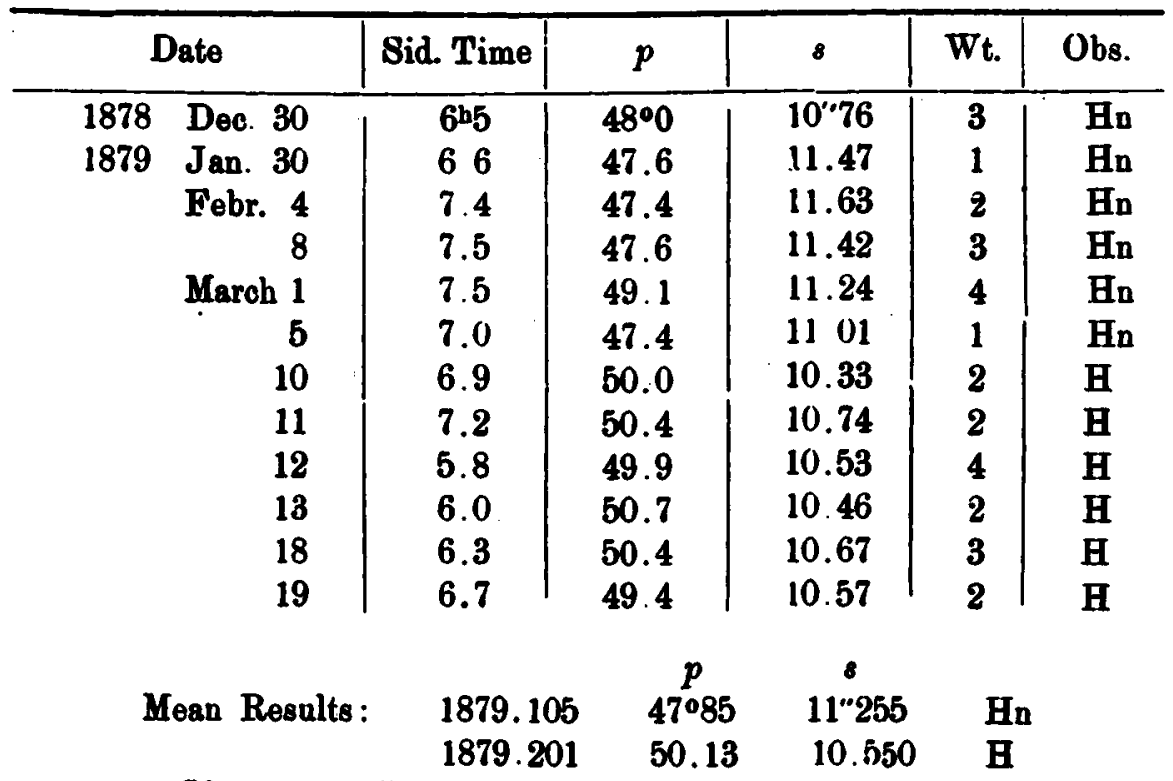

1879 , July 25

Observers: H, A. Hall; Hn, E. S. Holden. 